\title{
P02-5-26
}

Poster session

\section{Pathophysiological role of a factor affecting AGEs-RAGE interaction on endothelial cell responses}

\section{Shuji Mori ${ }^{1}$, Masahiro Watanabe ${ }^{1}$, Hidenori Wake ${ }^{2}$, Keyue Liu², Kiyoshi Teshigawara ${ }^{2}$, Hideo Takahashi ${ }^{3}$, Masahiro Nishibori ${ }^{2}$, Takao Toyomura ${ }^{1}$}

${ }^{I}$ Department of Pharmacology, School of Pharmacy, Shujitsu University, Japan, ${ }^{2}$ Department of Pharmacology, Graduate School of Medicine, Dentistry and Pharmaceutical Sciences, Okayama University, Japan, ${ }^{3}$ Department of Pharmacology, Faculty of Medicine, Kindai University, Japan

Objective: Advanced glycation end products (AGEs) are generated via Maillard reaction, in which non-enzymatic glycation occurs between reducing sugars (or their metabolites) and amine residues on biomolecules. AGEs stimulate vascular cells or macrophages through RAGE signaling pathway, and are involved in the exacerbation of tissue remodeling and the prolongation of inflammation. Therefore, it is likely that AGEs-RAGE signaling via RAGE stimulation is an important therapeutic target for the prevention or control of tissue remodeling and chronic inflammatory diseases.

Design and Methods: In order to clarify the pathophysiological significance of AGEs, we performed the comprehensive search of the factor exhibiting high affinity for HMGB1, another RAGE ligand, by protein array analysis with biotinlabeled HMGB1 as specific probe. In addition, the effect of HMGB1-binding factor on in vitro AGEs-RAGE binding, and on AGEs- induced cellular response using endothelial cell line were investigated.

Results: By the array analysis, we found the factor belongs to TNF superfamily showed the high affinity for HMGB1. This HMGB1-binding factor also influenced the in vitro AGEs-RAGE interaction. Additionally, the studies using endothelial cell line revealed that the IL- 8 mRNA expression was significantly increased by HMGB1-binding factor, and this enhancement of IL-8 mRNA level was abolished by the co-addition of AGEs.

Conclusion: The HMGB1-binding factor suggested to be involved in the formation of tissue remodeling and chronic inflammation by interaction with AGEs. 